\title{
The Historical Activity of Comet 55P/Tempel-Tuttle and Large Leonid Meteoroids
}

\author{
Martin Beech \\ Campion College and The Department of Physics, The University of \\ Regina, Regina, Saskatchewan, Canada S4S 0A2
}

\begin{abstract}
Even though comet 55P/Tempel-Tuttle has an orbital period of about 33 years it has only been recovered five times in the past 630 years. The earliest clearly documented return is that of 1366 , with the others being in 1699, 1865, 1965 and 1998. The comet may have been briefly sighted in 1035 (indicative of a possible outburst) and in 1234 and it was conspicuous by its non-recovery in 901 (possibly indicating very low surface activity during that return). We review the absolute magnitude data for comet 55P / Tempel-Tuttle and find tentative evidence to suggest it underwent an outburst in 1699. If large meteoroids were ejected from the comet during the 1699 outburst numerical integration studies find that they would have been Earth-orbit crossing in 1832 and 1965 - years in which the Leonid shower was rich in bright fireballs. The Earth will also sample 1699 ejected material in November 2001.
\end{abstract}

\section{Introduction}

Even though comet 55P/Tempel-Tuttle has an orbital period of about 33 years it has only been recovered five times in the past 630 years. The earliest documented return of $55 \mathrm{P} /$ Tempel-Tuttle is that of 1366 , when Chinese texts described the appearance of a "sparkling star" having the colour of "loose cotton" (see e.g. Kronk 1999). The other detected returns of the comet were in 1699, 1865, 1965 and 1998.

Yeomans et al. (1996) have studied the orbital evolution of comet 55P/Tempel-Tuttle in some considerable detail, and find possible, but not conclusive, evidence for Oriental sightings of the comet in 1035 and 1234. Interestingly, Yeomans et al. (1996) note that for 55P/Tempel-Tuttle to have attained nakedeye visibility in 1035 it must have been anomalously bright - that is, the comet may have undergone an outburst during its 1035 return to perihelion. Yeomans et al. (1996) also note that it is surprising that the comet was not recovered (or at least no records have as yet been found) during its very close and favourable approach to Earth in 901 . While the historical data is far from complete there does appear to be some indication that comet 55P/Tempel-Tuttle has undergone variations in its activity. Specifically, the comet may have been undergoing outbursts in 1234 and 1699 (see below) and it may have been nearly inert in 901. 


\section{The Leonid meteor shower}

A striking similarity between the orbital parameters of comet $55 \mathrm{P} /$ TempelTuttle and the November (now Leonid) meteoroid stream was first noted by Carl F.W. Peters in 1867 (Hughes 1982). The earliest traceable record of the Leonid shower dates back to AD 902, when a shower of "small star like fires" was observed in Sicily (Dall'Olmo 1978). Perhaps the most outstanding observational characteristic of the Leonid shower, however, relates to the large number of storms (when the visual meteor count exceeds 1000 per hour) that it has produced. Indeed, the Leonid shower accounts for 12 of the 26 meteor storms that have been recorded during the past one thousand years (Beech, Brown, \& Jones 1995).

While the Leonid meteor shower is active at some small level each year, it typically displays enhanced meteor rates, at the time of shower maximum, when 55P/Tempel-Tuttle is near perihelion. Yeomans (1981) has shown that the most likely circumstances under which a Leonid storm might materialize are when the Earth cuts through the stream inside of the comet's orbit at a time one to two years after the comet has passed perihelion. The picture is complicated, however, by the stream suffering occasional gravitational perturbations by the planet Jupiter (Brown \& Jones 1996). Strong jovian perturbations of the stream, for example, resulted in the less than spectacular Leonid display of 1899 (Brown 1999). Asher (1999) and Asher et al. (1999) have also demonstrated that a 5:14 jovian resonance can lead to the existence of long-lived streamlets of Leonid meteoroids. Indeed, it is believed that the 1998 Leonid storm was the result of the Earth intercepting the Leonid streamlet composed of meteoroids ejected from comet 55P/Tempel-Tuttle in 1333 (Asher et al. 1999).

Modern research has clearly demonstrated that it is a complex and nontrivial exercise to model the orbital evolution of Leonid meteoroids. The historical record of meteor showers, however, has proved invaluable in unraveling the circumstances under which Leonid storms might occur. Below, however, we wish to explore another aspect of Leonid stream structure, and this relates to the possible existence of large, meter-sized objects within the stream. The historical identification of large objects within the Leonid stream would be very interesting in that their presence potentially provides some insight into the outburst activity and nuclear structure of comet 55P/Tempel-Tuttle.

\section{Cometary outbursts and large meteoroids}

It has been clear for many years now that cometary nuclei are predominantly composed of various ices with an admixture of organic molecules and non-volatile inclusions. The basic "dirty snowball model" was first outlined by Whipple (1950), but variants on the original theme have arisen in recent years. While the predominantly icy nature of cometary nuclei is maintained in all contemporary comet models, the variant feature typically relates to the way in which the nucleus is assembled. Rather than the nucleus being a solid, homogeneous structure, it is now thought that the typical cometary nucleus is a conglomerate 'rubble pile' in which ice boulders of varying size are held together by their mutual gravitational attraction and an ice matrix 'glue'. Within the framework 
of the conglomerate nucleus models one can envisage the placement of large ice boulders into a meteoroid stream through the action of nuclear fragmentation, mantle loss events and cometary outbursts. Beech \& Nikolova (2000) have determined that the typical lifetime against sublimation for metre-sized ice fragments moving along orbits typical to that of meteoroid streams amounts to two to four perihelion passages. The rapid decay time of large ice fragments suggests that such objects will only rarely be found within meteoroid streams. This latter result follows since the typical time interval between cometary outbursts is likely to be equivalent to several tens of perihelion passages. With respect to comet 55P/Tempel-Tuttle we have but limited data to deal with and its outburst rate is not known with any precision.

The activity of a comet may be gauged according to its derived absolute magnitude $H$, where $H$ corresponds to the comet's apparent visual magnitude if it were placed $1 \mathrm{AU}$ from both the Earth and the Sun. Hughes (1987) has shown that the absolute magnitude can be related to the size of the cometary nucleus $(R)$ and the fraction $(f)$ of the comets surface that is undergoing active sublimation. The formula derived by Hughes gives, $\log (R / \mathrm{km})=1.114-0.5 \log f-0.2 H$. If one interprets any observed change in $H$ due to variations in the activity fraction $f$ (that is, if one assumes that from one perihelion return to the next the radius $R$ does not undergo any appreciable change), then in principle one can attempt to unravel the activity history. A small absolute magnitude implies a large value of $f$, and hence an active comet. A large value of $H$ implies a small value of $f$, and an inactive cometary nucleus. The absolute magnitude data for $55 \mathrm{P} /$ Tempel-Tuttle during its five recovered perihelion passages has been reviewed by Beech, Hughes, \& Murray (2000) and they find tentative evidence to suggest that the comet underwent an outburst during its 1699 return to the inner Solar System. Indeed, the estimated absolute magnitude of the comet in 1699 was two magnitudes brighter than that derived in 1998 . On the basis that 55P/Tempel-Tuttle did undergo an outburst or mantle loss event in 1699 we have numerically followed the orbital evolution of 1000 hypothetical large meteoroids (with masses of $10 \mathrm{~kg}$ ) placed in the Leonid stream at a time when the comet was near perihelion. The numerical procedure includes the effect of planetary perturbations and is similar to that described in Beech, Brown \& Jones (1996). Provided that the separation velocity between the nucleus and the meteoroids was greater than $3 \mathrm{~m} \mathrm{~s}^{-1}$ it is found that the fragments evolve on to Earth crossing orbits. It was also found that the Earth could theoretically have sampled some of the hypothetical objects ejected in 1699 during the Leonid returns of 1832 and 1965. Both the 1832 and 1965 Leonid storms were rich in bright fireballs (Brown 1999), and this clearly indicates the presence of large meteoroids.

\section{Leonid storms and large meteoroids}

One of the greatest Leonid storms in history is that of November 1833. It has been estimated that the peak hourly rate of visual meteors amounted to about 60,000 (Brown 1999). The 1833 Leonid storm, however, is perhaps all the more interesting for the reports of electrophonic sounds. Keay (1993) has described the circumstances under which sustained electrophonic sounds might be heard, 
and Beech (1998) has used Keay's model to argue that the threshold size for a Leonid meteoroid to produce sustained electrophonic sounds is of order one metre in diameter. The most likely means by which objects a metre or more in diameter could be deposited in the Leonid stream is through nuclear fragmentation or mantle loss episodes. Meteoroids of this large size can not be 'lifted' from the nucleus of $55 \mathrm{P} /$ Tempel-Tuttle through coupling to the sublimation driven molecular gas outflow. Indeed, following the methods outlined by Beech \& Nikolova (1999), it is found that the largest meteoroids that might be ejected from the nucleus of comet 55P/Tempel-Tuttle, through coupling to the sublimation outflow, is some $35 \mathrm{~cm}$ in diameter. Asher (1999) suggests that the 1833 Leonid storm was the result of the Earth encountering material ejected from comet $55 \mathrm{P} /$ Tempel-Tuttle in 1800 . Unfortunately, we have no record of the comet's behaviour during the associated perihelion return.

\section{Discussion}

The historical record contains a wealth of data concerning the Leonid meteor shower and its parent comet 55P/Tempel-Tuttle. The historical record, however, is not complete and typically, when it does exist, it is not extensive. There is always the hope, however, that more records will be found, and this hope holds the promise for continued studies. If one dare have a wish list for further observations then, certainly, further accounts of comet $55 \mathrm{P} /$ Tempel-Tuttle would be welcome; did it truly undergo an outburst in 1699 , and was it really inactive in 901? Such questions can in principle be answered through the discovery of new chronicles. Likewise, are there more (or better) accounts of electrophonic sounds being heard during Leonid storms? Such observations, should they be found, could provide important data on the largest meteoroids that might occasionally reside within the Leonid stream.

\section{References}

Asher, D.J. 1999, MNRAS, 307, 919-924.

Asher, D.J., Bailey, M.E. \& Emel'yanenko, V.V. 1999, MNRAS, 304, L53

Beech, M. 1998, AJ, 116, 499

Beech, M., Brown, P. \& Jones, J. 1995, QJRAS, 36, 127

Beech, M., Brown, P. \& Jones, J. 1996, MNRAS, 283, 137

Beech, M. \& Nikolova, S. 1999, MNRAS, 305, 253

Beech, M. \& Nikolova, S. 2000, Planet. Space Sci. (in press)

Beech, M., Hughes, D. W. \& Murray, I. 2000, Earth, Moon \& Planets (submitted)

Brown, P. 1999, Icarus, 138, 287

Brown, P. \& Jones, J. 1996, in Physics, Chemistry and Dynamics of Interplanetary Dust, eds. Gustafson, B.A.S., \& Hanner, M.S. (San Francisco: PASP Conference Series), 113

Dall'Olmo, U. 1978, J. His. Astron., 9, 123 
Hughes, D.W. 1982, Vistas Astron, 26, 325

Hughes, D.W. 1987, Nature, 325, 321

Keay, C.S.L. 1993, J. Sci. Exploration, 7, 337

Kronk, G.W. 1999, in Cometography: A catalog of comets. Volume I. (Cambridge: CUP).

Whipple, F.L. 1950, ApJ, 111, 375

Yeomans, D.K. 1981, Icarus, 47, 492

Yeomans, D.K., Yau, K.K. \& Weissman, P.R. 1996, Icarus, 124, 407 